\title{
Lexical Rules for Deverbal Adjectives
}

\author{
Victor Raskin ${ }^{1}$ and Sergei Nirenburg \\ Computing Research Laboratory \\ New Mexico State University \\ Las Cruces, NM 88003 \\ \{raskin,sergei\}@crl.nmsu.edu
}

\begin{abstract}
This work belongs to a family of research efforts, called microtheories and aimed at describing the static meaning of all lexical categories in several languages in the framework of the MikroKosmos project on computational semantics. The latter also involves other static microtheories describing world knowledge and syntax-semantics mapping as well as dynamic microtheories connected with the actual process of text analysis. This paper deals with the microtheory of adjectival semantics in one specific aspect, namely, the optimization and facilitation of the lexical entries for deverbal adjectives with the help of lexical rules deriving such entries from those of the corresponding verbs. Deverbal adjectives turn out to be the largest single subclass in the adjective lexical category. Unlike scalar and relative adjectives, which are anchored in property and object concepts, respectively, in the underlying ontology, deverbal adjectives are based on process concepts. While acquisition with lexical rules is necessary, universal and efficient for the class of deverbal adjectives, our research shows that, often in spite of the strong appearance to the contrary, such acquisition is neither fully automatic nor cost-free. The work was based on the set of over 6,000 English and about 1,500 Spanish adjectives obtained from taskoriented corpora. The findings are largely language-independent, and only English examples are used throughout the paper.
\end{abstract}

\section{Introduction}

The general topic of this paper is the information about adjectival meaning which should be included in a computational lexicon. Having addressed this general topic elsewhere (Raskin and Nirenburg 1995), we focus here on a more specific topic of the meaning of deverbal adjectives and, even more specifically, on the lexical rules used to derive the meanings of such adjectives from those of the corresponding verbs.

An extensive literature review in Raskin and Nirenburg (1995: 3-20) has demonstrated a focus on a number of issues that the non-computational linguistics of adjectives has deemed important. These include adjective taxonomies, usually on the basis of more or less consistent external criteria, the dichotomy between predicating and non-predicating adjectives and the related dichotomy between qualitative and relative adjectives, the order of adjectives modifying the same noun, the degrees of comparison and scalability, and the substantivization of adjectives.

We have shown also (Raskin and Nirenburg 1995: 24-27) that computational lexicography and computational semantics impose totally different parameters of relevance on these issues. We discovered that developing adjective semantics for an application modifies many popular views on the subject. It becomes clear, for instance, that

- many adjectives do not modify semantically the nouns that they modify syntactically;

- adjectival (attributive) meanings may be delivered by other parts of speech, and thus the se-

\footnotetext{
${ }^{1}$ Also of Natural Language Processing Laboratory, Purdue University, West Lafayette, IN 47907.
} 
mantics of adjectives only partially reflects their possible syntactic distinctions;

- the major distinction among adjectives is scalar vs. non-scalar;

- the attributive/predicative distinction, dominating the current scholarship on the adjective, has virtually no semantic significance, thus essentially crushing any hope to derive meaning from deep syntactic analysis;

- there is a significant gap in our knowledge about relations between truly relative adjectives (as well as nominal modifiers in English) and the nouns they modify;

- the typology of scales for gradables emerges as the dominant issue in adjective semantics and lexicography.

Most importantly for this paper, the crucial taxonomic criterion for each adjective is its anchoring in the underlying ontology. Whether such an anchor is a property, object, or process concept defines the adjective as truly scalar, relative (denominal), or deverbal, respectively.

The existing literature on adjectives also shows a predictable scarcity of systematic semantic analyses or lexicographic descriptions of adjectives. The quantifier adjectives, being the closest natural language comes to formal logic, have been privileged in this respect--see, for instance, Jackendoff (1983), McCawley (1988: 594-630), Chierchia and McConnell-Ginet (1990: 406-430), Frawley (1992: 464-480). The second luckiest category is adjectives of measure, especially spatial and, to a lesser degree, temporal, which are also seen as being more logically structured (see Bierwisch 1967, 1989, Greimas 1966, Teller 1969, Zhurinskiy 1971, Dowty 1972, Siegel 1976: 107-149 and 1979, Spang-Hanssen 1990, Spejewski 1995, and others).

Obviously, the semantic analysis of adjectives shares many problems with the semantic analysis of anything in natural language. One specific problem, noted by very few scholars, is what Marx (1983--see also Marx 1977 and Szalay and Deese 1978) refers to as the "plasticity" of adjectival meaning, namely that the same adjective can emphasize a different property of a noun in a different context. Lahav (1989), working loosely in the Keenan and Faltz (1985) paradigm, presents the same property as the non-compositionality of adjectives. If, he argues, red birds, red houses, and red books mean all different kinds of redness--and they do--how can one derive the meaning of an Adj $N$ combination from the meaning of the adjective and the noun? In other words, each noun, he believes, influences the meaning of the adjective. Katz (1972: 752), analyzing the meaning of good, is virtually the only author to have come up with a specific, even if definitely not complete account of how this works. Certain classes of nouns, he asserts, offer specific properties for good to work on:

"[t]he respects in which evaluations of things can be made differ with differences in the other semantic features of the words that refer to those things." (Artifact) permits evaluation of uses; (Component of a system) functions; (Role) duties; (Ornamentation) purposes; (Food) pleasurability and healthfulness; there are many others."

This situation, in which semantic analyses and lexicographic descriptions of adjectives (and other categories) are rare, is bound to change rapidly. As computational semantics moves to large-scale systems serving non-toy domains, the need for large lexicons with entries of all lexical categories in them is becoming increasingly acute, and the attention of computational semanticists and lexicographers is turning more towards such previously neglected or avoided categories as the adjectives. Recently, there have appeared some first indications of this attention--see, for instance, Smadja (1991), Beckwith et al. (1991), Bouillon and Viegas (1994), Justeson and Katz (1991, 1995), Pustejovsky (1995: 20-23). This research is a step in the same direction. 


\section{The Ontology-Based Approach to Adjectival Semantics and Lexicology}

In this section, we briefly review the basis of our approach to adjectival meaning and illustrate it on three examples of adjectival lexicon entries, i.e., one each for the three major classes of adjectives.

\subsection{The Ontological Approach}

Our work on adjectives forms a microtheory used by the MikroKosmos semantic analyzer. The architecture of MikroKosmos is described in Onyshkevych and Nirenburg 1994 and Beale et al. 1995. The MikroKosmos project is a component of a knowledge-based machine translation system (see Nirenburg et al. 1992). The purpose and result of the MikroKosmos analysis process is a rendering of the source language text into an interlingua text. The interlingua language is called the "text meaning representation" (TMR) language, and the TMR of a text is its representation in this particular type of interlingua. TMRs are realized in a frame-based language, where frame names typically refer to instances of ontological concepts and slots are usually filled with values of properties of those concepts. An ontology is, thus, a necessary prerequisite for building a TMR language.

"An ontology for NLP purposes is a body of knowledge about the world (or a domain) that a) is a repository of primitive symbols used in meaning representation; b) organizes these symbols in a tangled subsumption hierarchy; and c) further interconnects these symbols using a rich system of semantic and discourse-pragmatic relations defined among the concepts" (Mahesh and Nirenburg 1995: 1). The function of the ontology is to supply "world knowledge to lexical, syntactic, and semantic processes" (ibid).

The lexicon in MikroKosmos "mediates between the TMR and ontology" (Onyshkevych and Nirenburg 1994: 2). Lexicon entries for most open-class lexical items represent word and phrase senses, which can be either directly mapped into ontological concepts or derived by locally (that is, in the lexicon entry itself) modifying constraints on property values of concepts used to specify the meaning of the given lexical item. In the following section, we briefly illustrate the structure of those parts of the lexicon entry in MikroKosmos which bear on the description of the three types of adjectival meaning, scalar, denominal, and deverbal.

\subsection{The Ontological Approach to the Meaning of Adjective Types}

\subsubsection{Scalar Adjectives}

Our microtheory associates the meaning of a typical truly scalar adjective with a region on a scale which is defined as the range of an ontological property. The contribution that the adjective makes to the construction of a semantic dependency structure (TMR) typically consists of inserting its meaning (a property-value pair) as a slot in a frame representing the meaning of the noun which this adjective syntactically modifies.

Thus, in big house, big will assign a high value as the filler of the property slot SIZE of the frame for the meaning of house (see also Raskin and Nirenburg 1996). (1) is a partial lexical entry for big, with just two of the 13 lexical zones represented:

(1) (big (big-Adj1

(CAT adj) 


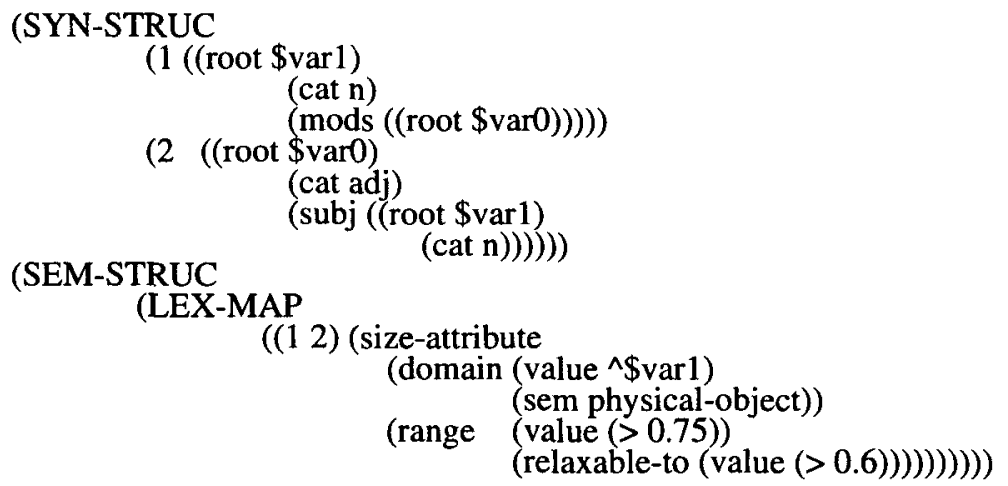

SIZE is an ontological concept of the SCALAR-PHYSICAL-OBJECT-ATTRIBUTE-PROPERTY type, with the term 'scalar' used here, as it is customarily, primarily in the sense of 'gradable.' Big is, of course, a typical gradable adjective, and as such, has a numerical scale associated with it the microtheory. Each numerical scale can be measured in actual measuring units, such as linear-size in feet, yards, or millimeters, or time in seconds. But often natural language expressions do not refer to absolute magnitudes but rather to abstract relative ones, as in the case of big. We assume a 0 to 1 numerical range for such abstract scales. For abstract references to size, the fillers in English can be:

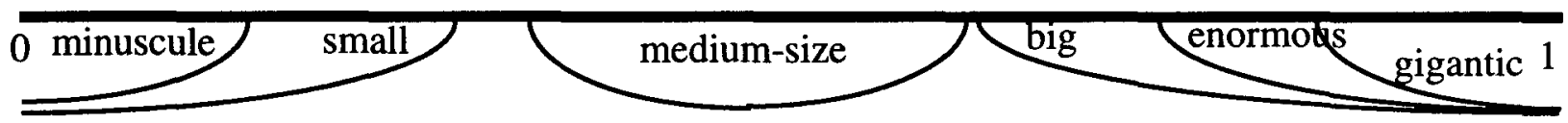

Big will, then, get something like a ' $>0.75$ ' value on the SIZE scale (1). These values are a crucial part of the lexical mapping (LEX-MAP) included in the semantics (SEM-STRUC) "zone" of a scalaradjective lexical entry. ${ }^{2}$.

Equally crucial is the syntactic-semantic mapping between the syntactic-structure (SYN-STRUC) and SEM-STRUC zones with the help of special variables. In the former, there are two subcategorization patterns, marked 1 and 2, listed in SYN-STRUC of (1). The former pattern corresponds to the attributive use of the adjective: the noun it modifies is assigned the variable \$var 1 , and the adjective itself the variable $\$ v a r 0$ in the modifier position. The latter pattern presents the noun, bound to \$var1, in the subject position and the adjective in the predicative position. In the SEM-STRUC zone, instead of variables which are bound to syntactic elements, the meanings of the elements re-

\footnotetext{
${ }^{2}$ Similar values are assigned to any scale based on a property concept. It should be noted, however, that numerical values like these correspond to the feature of gradability, which extends beyond scalarity: all scalar adjectives are gradable but not all gradable adjectives are true scalars. The two features are typically confused in the literature. This is why we distinguish here between true scalars, i.e., those adjectives whose meanings are based on a scale that is a property concept in the ontology, and all the other adjectives which are gradable and may be also loosely referred to as "scalars." Thus, many denominal and deverbal adjectives, in fact, most adjectives, can form a comparative or superlative degree form with the help of more or most ___, respectively, e.g., more medical (3ii), more aeronautical (4), more employable (6), more abusive (9-10). All of these adjectives are, therefore, gradable. Now, none of them is anchored in a scale-type property concept, however, which makes them all, technically, non-scalars but, in more practical and customary terms, non-true scalars.
} 
ferred to by these variables (and marked by a caret, '^’) are used. That is, $\wedge \$$ var1 reads as "the meaning of the element to which the variable \$var1 is bound." Among the constraints listed in the SEM-STRUC zone of an entry, are selectional restrictions (the noun must be a physical object) and relaxation information, which is used for treatment of unexpected ("ill-formed") input during processing.

Thus, an entry like (1) should be read as follows:

- first, the entry is the specification of a single sense, while the superentry is the set of such entries;

- the second line assigns a sense number to the entry;

- next, the adjective is assigned to its lexical category;

- the first subcategorization pattern in the SYN-STRUC zone describes the Adj-N construction; the second subcategorization pattern describes the N-Copula-Adj construction;

- the LEX-MAP part of the SEM-STRUC zone defines the lexical semantics of the adjective by assigning it to the class of SIZE adjectives, that is, anchoring it in the ontological propertytype concept SIZE and stating that it is applicable to physical objects and that its meaning is a high-value range on the SIZE scale/property.

In the case of continuous scales, like SIZE, the acquisition of all the adjectives served by this scale is greatly facilitated and expedited after the first one of them gets a lexical entry: each new adjective needs only an appropriate range assigned to it, and the rest of the information in the semantic zone of the entry, as indeed in all the other zones as well, remains the same. We will recall this methodological circumstance shortly, in Section 3.

\subsubsection{Denominal Adjectives}

The most general case of a denominal adjective-entry and its connection to that of the corresponding noun is demonstrated in (3):

(3)

(i) (medicine (medicine-N1)

(CAT n)

(SYN-STRUC

(root \$var0)

(SEM-STRUC (cat $n))$ )

(LEX-MAP

(ii)

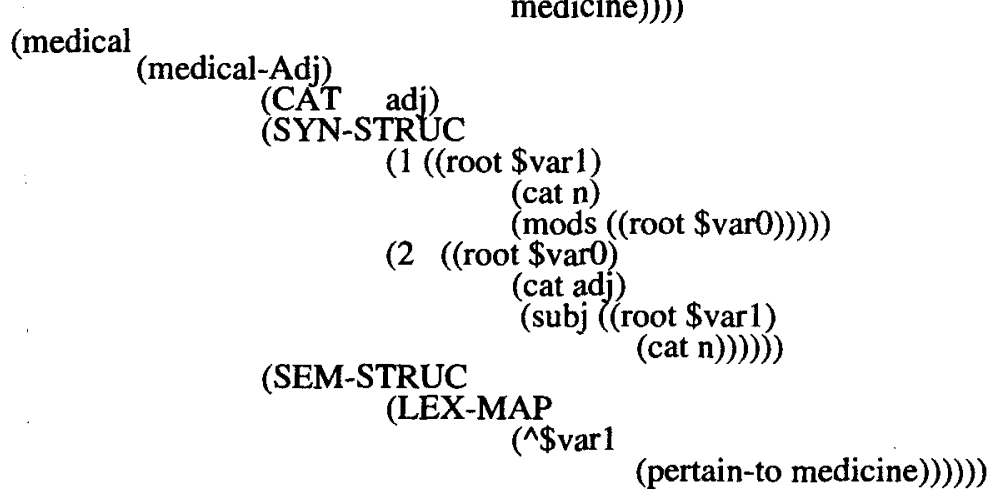

The formula of transition from the noun entry to that of the adjective is simple and transparent, and it remains constant for this type of adjective. In Raskin and Nirenburg (1995: 39-41), we discuss the desirability, feasibility, and usefulness of replacing the catch-all "pertain-to" dependency 
with a more specific and informative one, and while we demonstrate several of those, we argue for staying at this generic level of granularity in a significant number of cases.

Denominal adjectives are relative, and, as such, are expected to be non-scalar and non-predicating. A true relative adjective cannot indeed be used predicatively and or comparatively, but, practically, it is hard to come up with an example which is guaranteed against that. After all, (4i-ii) are not really ill-formed, and it is hard to imagine a more truly-relative adjective than aeronautical 'related to aeronautics.'

(4) (i) His approach to the problem was aeronautical.

(ii) His approach to the problem was much more aeronautical than mine.

Clearly, a productive semantic process, a shift, takes place here, probably along the lines of (5), and, therefore, a dynamic rule exists which creates adjective entries for these predicating, pseudoscalar, pseudo-qualitative senses of the seemingly perfectly relative adjectives. Their one telling difference from the truly qualitative, predicating, scalar adjectives is that the relative adjectives cannot make the qualitative shift in the attributive position.

(5) Pertaining to [noun meaning] --->Pertaining more to [noun meaning]

The difficulty of finding a relative adjective which would be absolutely resistant to a predicative shift of meaning is similar to the difficulties Katz (1972: 753) had, looking for a noun which could never be used with good.

\subsubsection{Deverbal Adjectives: First Glimpse}

A large class of adjectives whose meanings are derived from those of verbs also straddle the scalar / non-scalar divide. The event-related scalars (actually, gradables--see fn. 2) do not really differ from true scalars in terms of their gradability (6); the event-related non-scalars can acquire gradability at the cost of a meaning shift or marginal acceptability (7).

(6) (i) Jake is employable

(ii) Jake is very employable

(iii) Jake is more employable than Bob

(iv) Jake is most employable of all

(v) Bob is barely employable

(i) ?Jake's initiative was abortive

(ii) 'Jake's initiative was very abortive

(iii) 'Jake's initiative was more abortive than Bob's

(iv) 'Jake's initiative was most abortive of all

(v) 'Bob's initiative was barely abortive

Examples (8-10) illustrate a typical transition from the lexical entry of a verb to those of the deverbal adjectives. Both the verb entry and the adjective entries are much more complex than those for nouns and denominal entries.

$$
\text { (abuse (abuse-V1 }
$$


(9)

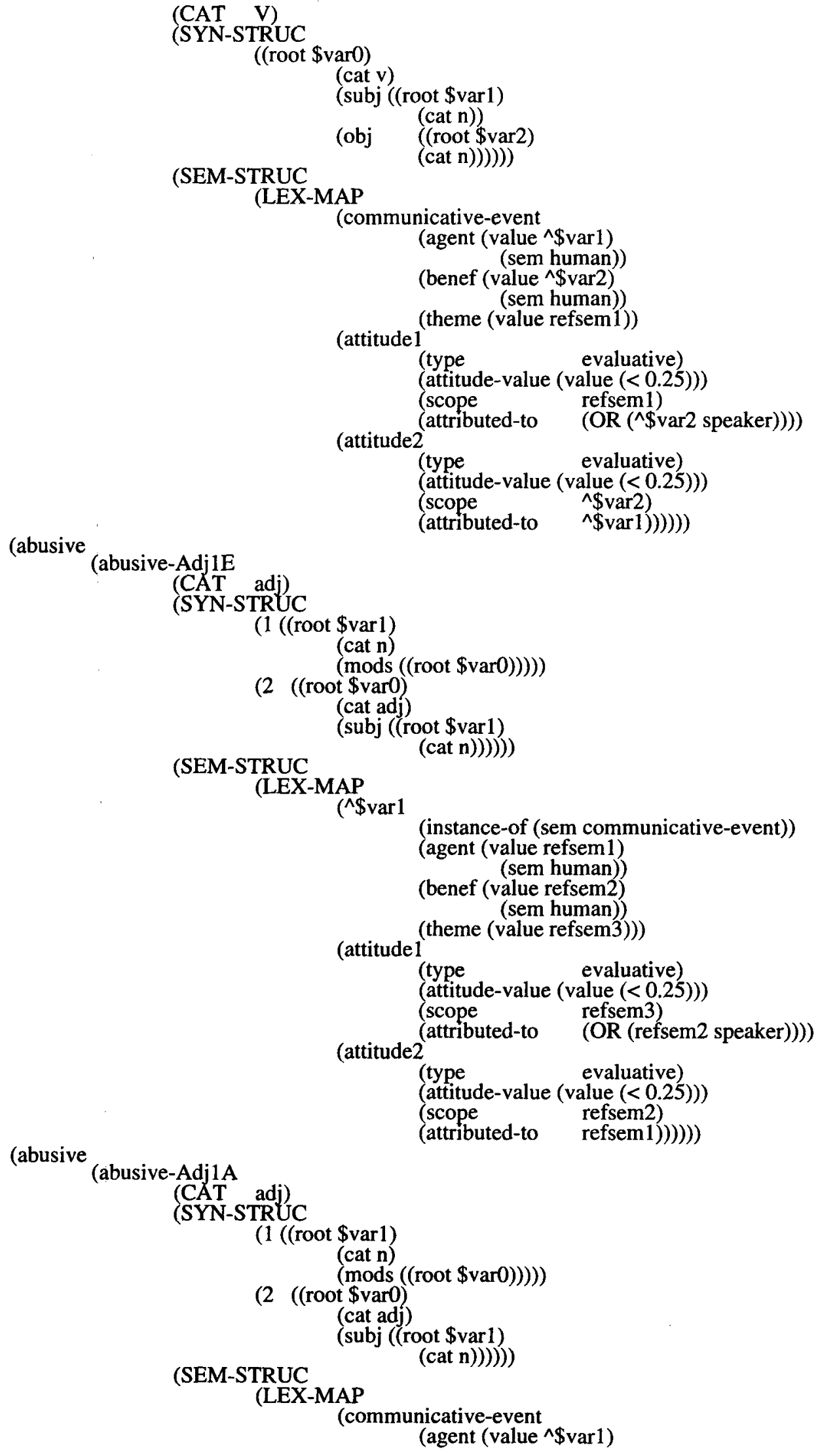

(10) 


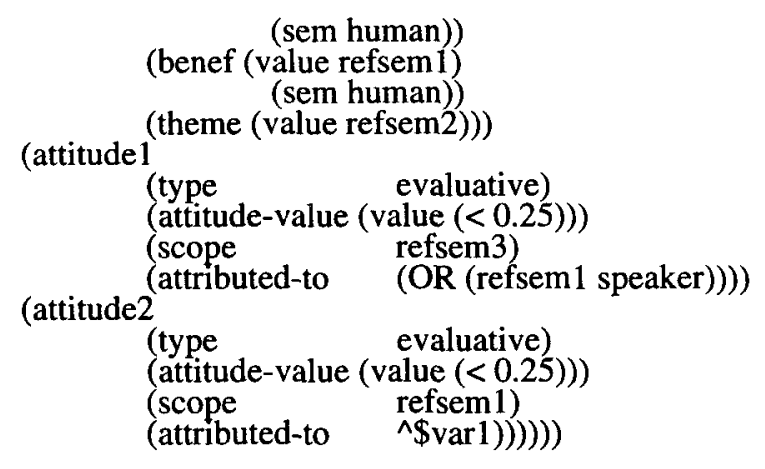

This sense of the verb, "insult verbally," is anchored in the process concept COMMUNICATIVEEVENT, modified by two evaluative attitudes, involving two unmapped (i.e., internal for the zone) variables, refsem 1 and refsem 2 . Attitude 1 means that the beneficiary or. more appropriately, the victim, of the process, i.e., the abused person and/or the speaker evaluate(s) the theme of the abuse, i.e., the contents of what is said, as bad; attitude2 means that the agent of abuse-VI thinks of the beneficiary pretty poorly.

To derive the semantics zone of an adjectival entry from that of the corresponding verbal entry, one must first identify the case, or thematic role (such as agent, theme, beneficiary, etc.) filled by the noun modified by the adjective in question. What is abusive is either the event (E) itself, as in abusive speech or abusive behavior, or the agent (A) of the event, as in abusive man or abusive neighbor. Abusive ${ }_{I E}(9)$ is then the eventive sense of the adjective formed from abuse-VI (8), and abusive $_{I A}(10)$ is the agentive sense of the adjective in the same sense of abuse. There may, in fact, be at least 4 more senses, and entries, for the adjective, but we will come back to them shortly, in Section 4.1.

\section{Lexical Rules}

In this section, we introduce our notion of lexical rules (LRs), place their origin in the descriptive methodology and heuristics of large-scale lexical acquisition, and establish some usability parameters for them.

The "transition formulae" from a noun lexical entry (3i) to that of a denominal adjective (3ii) or from a verb lexical entry (8) to those of deverbal adjectives (9-10) are examples of LRs in which we are interested here. Each LR takes a ready entry $\left(E_{1}\right)$ and creates another entry $\left(E_{2}\right)$ out of it automatically:

$$
\operatorname{LR}\left(E_{1}\right)=E_{2}
$$

Typically, each $\mathrm{E}_{1}$ is produced "manually," that is, by a qualified human on the basis of all the available heuristics for the lexical acquisition process (see Raskin and Nirenburg 1995: 41-57). These include methods of reducing unnecessary polysemy, gauging the optimal grain size, and determining the meaning, not the least of which is selecting the ontological concept in which the entry is anchored. These methods are, of course, captured in an array of semi-automatic tools of ontological and lexical acquisition developed for the MikroKosmos project (see, for instance, Mahesh 1996 and Viegas and Raskin 1996).

These tools stemmed from a pilot project, in which templates for types of lexical entries were de- 
veloped truly manually and rapidly propagated on the basis of such obvious paradigmatic relations as synonymy and partial synonymy, antonymy, and hyper-/hyponymy, and much less obvious relations of partial overlap, such as exemplified above in connection with the lexical entry for big (1) and its easy adaptation to all the other SIZE adjectives by modifying the numerical value on the SIZE range (2), if necessary. The corpus of 6,000 English adjectives (an intersection of LDOCE and a corpus of 1987-99 Wall Street Journal articles) yielded some 250 SIZE adjectives, all of them propagated from the same template that underlies (1). The least trivial relationship to establish involved constrains on the modified noun, thus recognizing an adjective like well-endowed as characterizing the size of a certain part of human male anatomy, a different part of human female anatomy, or the amount of money available to an institution.

The discovery and use of such relationships and rapid propagation methods based on them lay the foundation for LRs. In fact, each such relationship and related method is a LR. Because we are engaged in massive lexical acquisition, we are obviously interested in LRs, which are:

- easily discoverable;

- massively productive;

- exception-free.

Perhaps the most easily discoverable LRs involve E1/E2 pairs which are morphologically related, especially if their share the roots. Some of these rules are transcategorial, i.e., E1 and E2 belong to different lexical categories. Obviously, the denominal adjective LR and the deverbal adjective LR (12i-ii, respectively), exemplified in (3) and (8-10), are precisely such LRs.

(i) $\quad \mathrm{LR}_{\mathrm{NA}}(\mathrm{N}$ entry $)=\operatorname{Adj}$ entry

ii) $\quad L R_{V A}(V$ entry $)=$ Adj entry

The additional attraction for using (12i-ii) in massive acquisition is the facilitation of the least trivial element of lexical semantic heuristics, namely, the discovery of the ontological concept on which the lexical entry should be based. While a relatively small class of true scalars is more or less easily associated with certain property concepts, there are many more process and object concepts, and it is much easier to relate the meaning of a verb or a noun to one of those. Besides, it is precisely the denominal and deverbal adjectives which are very hard to relate to a property concept directly, so the LRs come in quite handy.

Several of the LRs discovered manually in the pilot project turned out to be "large," in the sense of their massive productiveness (see, for instance, Viegas et al. 1996), such as the comparativedegree LR, applicable to every one of the 4,000-plus gradable adjectives. Given the paucity of relative adjectives in English (because of the adjectival use of nouns), the denominal adjective LR (12i) produces around 300 entries in our English corpus. The deverbal adjective LR falls in between, with an output of almost 1,000 entries.

We have found virtually no LR to be exception-free, and that reduces, of course, the degree to which a large LR can be used fully automatically, thus raising the cost of their application.

\section{Deverbal Adjectives: A Full(er) View}

In this section, we offer a bird's eye view of large-scale acquisition of deverbal adjectives with the help of $L_{V A}$, both in the general, common case, with its non-trivial complications, deviations, and exceptions, and in the particular case of the single largest and seemingly most regular subclass of 
deverbal adjectives, namely, those ending in -able/-ible. The review is conducted from the perspective of highly desirable full automation vs. the reality and unavoidability of exceptions requiring manual treatment.

\section{1 $\mathrm{LR}_{\mathrm{VA}}$ : Common Cases and Complications}

The common case of deverbal adjective acquisition includes most deverbal adjectives, and it is discussed first. The most statistically and conceptually significant complications, deviations, and exceptions from the common case are briefly sketched next.

\subsubsection{Common Case}

The 950 or so deverbal adjectives in our English corpus have received their entries as a result of the application of the deverbal adjective LR (12ii). The LR exists in at least these 6 forms, corresponding to the event or its semantic cases/thematic roles:

- event-itself (E), e.g., abusive in (9): abusive behavior;

- agent-of-event (A), e.g., abusive in (10): abusive husband;

- beneficiary-of-event (B), e.g., free in free bird;

- theme-of-event (T), e.g., automatic in automatic elevator;

- instrument-of-event (I), e.g., poisonous in poisonous food;

- location-of-event (L), e.g., international in international company.

As exemplified in (9-10), each case-related sub-LR operates in a well-defined way, placing ^\$var 1 in the SEM-STRUC zone in the value slot of the appropriate case of the event frame. Similarly, $\mathrm{LR}_{\mathrm{E}}$, the event-itself sub-LR places ${ }^{\wedge} \$$ var 1 in the event position. Obviously, then:

(i) $\quad \mathrm{LR}_{\mathrm{E}}$ (abuse-VI) $=$ abusive-1E(9)

(ii) $\quad \mathrm{LR}_{\mathrm{A}}$ (abuse-VI) $=$ abusive-1A (10)

Abuse has two more senses, abuse-V2 "violate a law or a privilege" and abuse-V3 "assault physically." Abusive can inherit all of those senses, both in the eventive and agentive varieties, thus adding 4 more senses to its superentry. Abusive is indeed a pretty typical and "easy" example of LR VA $_{\text {. }}$ Even so, there are several aspects of its application which require human judgment.

\subsubsection{Selective Semantic Cases}

The first obvious complication is that not all of its semantic cases of a verb are usable for the LR-in fact, only one of the three semantic cases of abuse, namely, agent is but beneficiary and theme are not. This throws the first monkey's wrench into making the LR fully automatic. Here, as in many similar cases in side and outside of this LR, we face a dilemma. We can either try and discover a rule which marks some semantic cases as participating in the LR and others as not participating in it. Such a principle is not immediately clear and may:

- not exist at all;

- be very hard to research;

- be cumbersome and involve a complicated procedure;

- be exception-ridden, thus coming with a possibly lengthy list or several different lists;

- be not machine-tractable.

The other alternative is to check each verb manually, i.e., by a qualified human. We will see that 
there are other factors which push this latter decision forward.

\subsubsection{Selective Meanings in Polysemous Verbs}

It is interesting to note that the sense distinctions in abusive seem to be less significant than in abuse. Typical of many cases of polysemy, this may be an indication that the distinctions in the dictionaries may be disposable (see the Spanish verb dejar reduced from 52 dictionary meanings to 7 in Nirenburg et al. 1995). Perhaps less so in the case of abuse than in the case of abandon directly below, it is reasonable to suggest that, in many cases of dictionary polysemy, it is the single sense of the verb modified by different types of nouns that can fill its case slots. Such cases are candidates for reducing polysemy as part of the heuristics procedures mentioned above (see also Raskin and Nirenburg 1995: 41-45).

A human must decide whether the adjective inherits all the senses of a polysemous verb because it is not always the case that it does--unlike abusive. Thus, impregnable has only the figurative sense of impregnate. Out of the 5 senses of abandon:

- abandon-V1: "stop trying" (aspectual);

- abandon-V2: "leave somebody or something at location";

- abandon-V3: "withdraw support";

- abandon-V4: "give up claim";

- abandon-V5: "yield to emotion"--

abandoned has 6 senses corresponding to just three of the senses (some examples below may have other meanings along with the one exemplified):

- abandoned-2B, e.g., abandoned friend;

- abandoned-2T, e.g., abandoned parcel;

- abandoned-3B, e.g., abandoned protégé;

- abandoned-3T, e.g., abandoned project;

- abandoned-4B, e.g., abandoned child;

- abandoned-4T, e.g., abandoned property--

and 1, possibly no longer existing sense corresponding to another sense of the verb:

- abandoned-5T, e.g., John was abandoned to his grief (theme is used here in the experiencer mode).

\subsubsection{Adjectives and Participles}

Abandoned can, of course, be a participle as well, but we have discovered no reason whatsoever to treat participles differently from deverbal adjectives. Surely, participles may have syntactic dependents and be used postpositively in English--more so than adjectives, but this is easily accounted for in the appropriate patterns (not demonstrated) in the adjective SYN-STRUC zone. An adjective, just as a participle, is a device to raise a proposition into a higher one. In fact, we suggest that all clearly participial adjectives must be treated exactly as we are proposing here, that is, with the help of $L_{\mathrm{VA}}$ : the only alternative is unfolding them into full-fledged clauses, and this is something MT should, and usually can, avoid dealing with--on the basis of the practical effability principle (Nirenburg and Raskin 1995: 45-47). That the difference between participles and adjectives may be a grammatical artifact--or that, at least, it is of no significance for computational semantics--can be indirectly but rather convincingly supported by the case of Russian, where the grammarians have been trying to prop up the distinction by insisting on the doubling of the spelling of $n$ in the suffix of the past participle but not of the related adjective, as in ranennyy $v$ ruku boetz "fighter wounded 
in the arm" and ranenyy boetz "wounded fighter": the speakers reject this distinction both in pronunciation, where no consonant doubling is detectable, and in spelling, where the two forms are universally confused.

\subsubsection{Accidental Gaps}

Cases like impregnable above raise another significant issue. The treatment of negation in deverbal adjectives is not very difficult: the negative quantifier can, in most cases, be simply added to the non-negative adjective, and this applies to all of the negative prefices, such as un-, in- (im-, etc.), dis-. But impregnable, like quite a few other negative deverbal adjectives, does not have a non-negative counterpart, which looks like an accidental gap. In fact, the non-existence of a deverbal adjective, positive or negative, from the physical meaning of impregnate "make pregnant" is also an accidental gap. And, of course, a further complication is that im-in the verb is not negative while in the adjective it is: this is, apparently, the reason for the non-existence of the positive adjective, but this reason is itself an accidental gap.

Gaps of this kind abound throughout the corpus. Why, for instance, there is perishable but not rottable. As we will see below, the former is a somewhat rare bird among the deverbal adjectives ending in -ble, but, on the face of it, there is no difference between food products rotting and food products perishing--in fact, the former sounds more natural than the latter. Nevertheless, rottable sounds less acceptable than perishable.

\subsubsection{Suppletivism}

Many deverbal adjectives in the corpus derive their lexical entries from verbs which are not morphologically related to them (14). The LR, however, works with the same ease as in typical, morphologically derivative cases. An even stronger case of suppletivism involves processes which do not have single-word verbs denoting them. Nevertheless, it often makes sense--and this is a human judgment--to construct such verbs out of the appropriate ontological concept with necessary constraints, because this is still the easiest way to construct lexical entries for some adjectives (15). Obviously, this kind of supersuppletivism would be impossible and arbitrary if implemented outside a justified situated ontology.

$\begin{array}{llll}\text { (i) } & \text { ablaze } & < & \text { burn } \\ \text { (ii) audible } & < & \text { hear } \\ \text { (iii) awful-2 } & < & \text { fear } \\ \text { (i) affectionate } & < & \text { show-affection } \\ \text { (ii) possible } & < & \text { can-happen } \\ \text { (iii) brainy } & < & \text { show-intelligence }\end{array}$

\subsubsection{Syncretism}

Related to suppletivism, we discover quite a few deverbal adjectives "shared" by several verbs on a synonymous sense. This, abandoned-2 is a deverbal of both abandon-V2 (see Section 4.1.3 above) and leave in the same sense of "leave somebody or something at location" because the latter's "own" deverbal, left, is not comfortable in adjectival use, especially attributively:

(16) (i) I abandoned a friend in Hope, AR--I have an abandoned friend in Hope, AR

(ii) I left a friend in Russell, KS--*I have a left friend in Russell, KS 


\subsection{Adjectives Ending in -ble}

A large subclass of deverbal adjectives are adjectives that end in able/ible (cf. Kjellmer 1986 and also Hall 1877, Jespersen 1942, Marchand 1960, Abraham 1970, Meus 1975, for a discussion of such adjectives in English, even though much of the discussion sheds little light on the semantic and lexicographic issues in hand). Just about all of them mean 'something than can be [verb]-ed': thus, readable means 'something that can be read.' In other words, a typical -able entry is derived from the lexical entry of the appropriate verb, with the positive potential attitude added, and in either the beneficiary or theme role, depending on the animateness/inanimateness of \$varl, respectively (17i-ii).

(i)

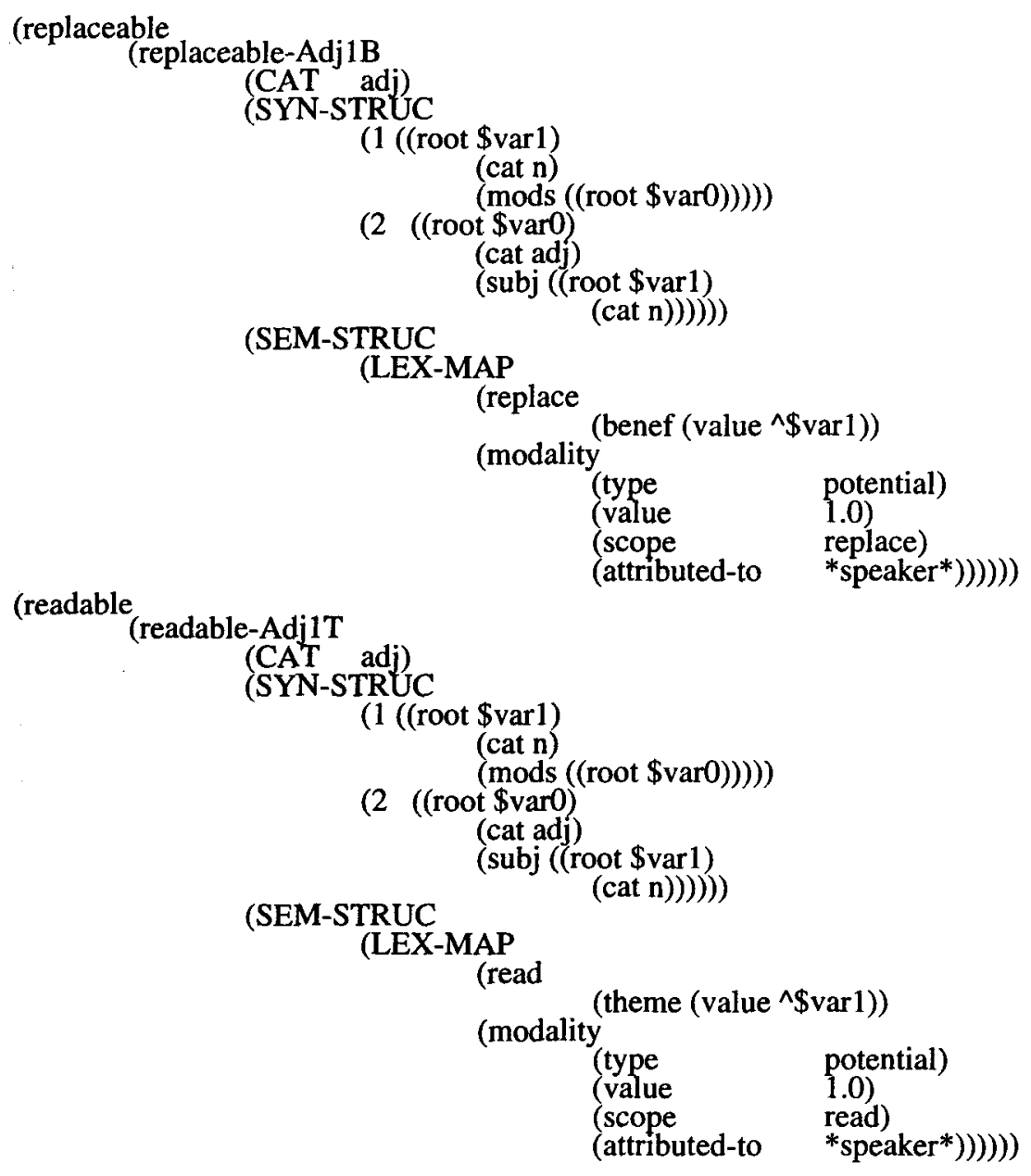

The modality in the entries above corresponds, of course, to 'can' in the informal formula above.

On the surface of it, these adjectives seem to be the best candidates for a fully automatic LR, especially if the beneficiary/theme distinction can be taken care of on the basis of the animateness or inanimateness of the modified--and this can be done. The problem is, however, that the beneficiary/theme combination of semantic cases for these deverbal adjectives, while accounting for a huge majority of such adjectives in the corpus (close to 270 out of the 300 or so), is only one of the 17 possible semantic-case combinations: 


\begin{tabular}{|c|c|c|c|}
\hline (ii) & A & culpable & $<d o-b a d$ \\
\hline (iii) & E & disreputable & $<$ feel-shame \\
\hline (iv) & $\mathrm{T}$ & comprehensible & $<$ understand -1 \\
\hline (v) & can $+\mathrm{A}$ & perishable & $<$ perish-2 \\
\hline (vi) & can $+A$ can $+E$ & audible & $<$ hear \\
\hline (vii) & can+B & acceptable-2 & $<$ accept -1 \\
\hline (viii) & can $+B$ can $+T$ & definable & $<$ define $-1-3$ \\
\hline (ix) & can $+B$ can $+T$ can $+A$ & adjustable & $<$ adjust $-1-2$ \\
\hline$(\mathrm{x})$ & $\operatorname{can}+\mathrm{L}$ & livable & $<$ live -1 \\
\hline (xi) & cant $\mathrm{T}$ & actionable & $<$ sue \\
\hline (xii) & not $+\mathrm{A}$ & incapable-2 & $<$ be-able \\
\hline (xiii) & not + can $+A$ & inviable & $<$ live -1 \\
\hline (xiv) & not+can+B & unacceptable-2 & $<$ accept-1 \\
\hline (xv) & not+can+B not+can+T & undefinable & $<$ define $-1-3$ \\
\hline (xvi) & not+can+L & uninhabitable & $<$ live -1 \\
\hline (xvii) & not+can $+T$ & impassable & $<$ pass $-1-4$ \\
\hline
\end{tabular}

Stripped of 'can' and 'not,' there are still 9 different types, and because 'can' and 'not' do not "apply" universally, their presence or absence are not trivial. Except for types (viii) and (xv), most of the types have few examples in the corpus, and perishable is alone in its type (v). What it means, again, is that human judgment is necessary in deciding which, if any, case form of $L_{V A}$ to apply to each verb.

\section{Conclusion}

In this paper, we have:

- introduced an ontological semantic approach to the lexical semantics of adjectives;

- described and exemplified two relevant zones from lexical entries of adjectives;

- demonstrated three major classes of adjectives, true scalars, denominals and deverbals;

- explained our notion of lexical rules and their importance to acquisition;

- discussed the lexical entries for deverbal adjectives and the role lexical rules play in their acquisition;

- focussed on the issue of full automation of lexical rules in large-scale acquisition;

- argued for a significant place for human judgment in the process.

We believe, therefore, that LRs are worth discovering and activating only if they are clearly massproductive, such as $L_{\text {VA }}$, which is central to this paper. Otherwise, the human cost of manual checking every verb entry before applying the rule to it would render each adjective entry obtained with the help of the LR more--or at least no less--expensive than if it were produced manually "from scratch."

Another important trade-off is between the cost of discovery and the productivity of the rule. It is pleasant and challengeable for a linguist to think that each pragmatic relation is worth discovering and activating in lexical acquisition. It is equally interesting to attempt to discover a rule for each and every exception and complication mentioned in Section 4. The Young-Grammarian approach to language was that every single fact had a rule attached to it. This is probably true but not practical. Neither is the current postmodern approach that there are nice rules and then an area of chaos "because language is that way." Our practical view is that a LR is useful for lexical acquisition if 
it is easily discoverable and very productive. We do not expect any such LR to be exception-free, and our methodology is comfortable dealing with those exceptions.

\section{Acknowledgment}

The research reported in this paper was supported by Contract MDA904-92-C-5189 with the U.S. Department of Defense. Victor Raskin is grateful to Purdue University for permitting him to serve as a consultant for the CRL/NMSU MikroKosmos project. Both authors feel indebted to the other members of the MikroKosmos team.

\section{References}

Abraham, Werner 1970. Passiv und Verbableitung auf e. -able, dt. -bar. Folia Linguistica 4, pp. 38-52.

Beale, Stephen, Sergei Nirenburg, and Kavi Mahesh 1995. Semantic Analysis in the Mikrokosmos Machine Translation Project. In Proceedings of the Second Symposium on Natural Language Processing (SNLP-95), August 2-4. Bangkok, Thailand.

Beckwith, Richard, Christiane Fellbaum, Derek Gross, and George A. Miller 1991. WordNet: A lexical database organized on psycholinguistic principles. In: Uri Zernik (ed.), Lexical Acquisition: Exploiting On-line Resources to Build a Lexicon. Hillsdale, N.J.: Erlbaum, pp. 211-232.

Bierwisch, Manfred 1967. Some semantic universals of German adjectivals. Foundations of Language 5:1, pp. 1-36. Bierwisch, Manfred 1989. Focussing on dimensional adjectives: Introductory remarks. In: Manfred Bierwisch and Ewald Lang (eds.), Dimensional Adjectives and Conceptual Interpretation. Berlin-Heidelberg: Springer-Verlag, pp. 1-11.

Bouillon, Pierrette, and Viegas, Evelyne 1994. A semi-polymorphic approach to the interpretation of adjectival constructions: A cross-linguistic perspective. In: Proceedings of the Sixth EURALEX International Congress, Amsterdam: Free University of Amsterdam, pp. 36-44.

Chierchia, Gennaro, and Sally McConnell-Ginet 1990. Meaning and Grammar: An Introduction to Semantics. Cambridge, MA-London: M.I.T. Press.

Dowty, David 1972. Temporally descriptive adjectives. In: John Kimball (ed.), Syntax and Semantics, Vol. I. New York: Seminar Press.

Frawley, William 1992. Linguistic Semantics. Hillsdale, N.J.: Erlbaum.

Greimas, A. J. 1966. Sémantique structurelle. Paris: Larousse.

Hall, Fitzedward 1877. On English Adjectives in -ABLE with Special Reference to RELIABLE. London: Trübner. Jackendoff, Ray 1983. Semantics and Cognition. Cambridge, MA-London: M.I.T. Press.

Jespersen, Otto 1942. A modern English Grammar on Historical Principles. Part 6: Morphology. Copenhagen: Munksgaard.

Justeson, John S., and Slava M. Katz 1991. Co-occurrences of antonymous adjectives and their contexts. Computational Linguistics 17:1, pp. 1-19.

Justeson, John S., and Slava M. Katz 1995. Principled disambiguation: Discriminating adjective senses with modified nouns. Computational Linguistics 21:1, pp. 1-27.

Katz, Jerrold 1972. Semantic theory and the meaning of good. Journal of Philosophy 61, pp. 736-760.

Keenan, Edward L., and L. M. Faltz 1985. Boolean Semantics for Natural Language. Dordrecht-Boston: D. Reidel. Kjellmer, Göran 1986. Legible but not readable: On the semantics of English adjectives in -ble. Studia Neophilologica 58:1, pp. 11-38.

Lahav, Ran 1989. Against compositionality: The case of adjectives. Philosophical Studies 57, pp. 261-279.

Mahesh, Kavi 1996. Ontology Development for Machine Translation: Ideology and Methodology. Memoranda in Computer and Cognitive Science MCCS-96-292. Las Cruces, N.M.: New Mexico State University

Mahesh, Kavi, and Sergei Nirenburg 1995. A situated ontology for practical NLP. A paper presented at the IJCAI ' 95 Workshop on Basic Ontological Issues in Knowledge Sharing. Montreal, August 19-21.

Marchand, Hans 1960. The Categories and Types of Present-Day English Word-Formation. Wiesbaden.

Marx, Wolfgang 1977. Die Kontextabhängigkeit der Assoziativen Bedeutung. Zeitschrift für experimentelle und angewandte Psychologie 24, pp. 455-462.

Marx, Wolfgang 1983. The meaning-confining function of the adjective. In: by Gert Rickheit and Michael Bock (eds.), Psycholinguistic studies in language processing. Berlin: Walter de Gruyter, pp. 70-81.

McCawley, James D. 1988. The Syntactic Phenomena of English, Vol. II. Chicago: Chicago University Press.

Meus, V. 1975. English adjectives in -able. Studia Germanica Gandensia 16, pp. 35-59. 
Nirenburg, Sergei, Jaime Carbonell, Masaru Tomita, and Kenneth Goodman 1992. Machine Translation: A Knowledge-Based Approach. San Mateo, CA: Morgan Kaufmann.

Nirenburg, Sergei, Victor Raskin, and Boyan Onyshkevych 1994. Apologiae ontologiae. Memoranda in Computer and Cognitive Science MCCS-95-281. New Mexico State University: Computing Research Laboratory. Reprinted in: Judith Klavans, Bran Boguraev, Lori Levin, and James Pustejovsky (eds.), Symposium: Representation and Acquisition of Lexical Knowledge: Polysemy, Ambiguity, and Generativity. Working Notes. AAAI Spring Symposium Series. Stanford, CA: Stanford University, 1995, pp. 95-107. Reprinted in a shortened version in: TMI 95: Proceedings of the Sixth International Conference on Theoretical and Methodological Issues in Machine Translation. Centre for Computational Linguistics, Catholic Universities Leuven, Belgium, 1995, pp. 106-114.

Onyshkevych, Boyan, and Sergei Nirenburg 1994. The lexicon in the scheme of KBMT things. Memoranda in Computer and Cognitive Science MCCS-94-277. Las Cruces, N.M.: New Mexico State University.

Pustejovsky, James 1995. The Generative Lexicon. Cambridge, MA: MIT Press.

Raskin, Victor, and Sergei Nirenburg 1995. Lexical semantics of adjectives: A microtheory of adjectival meaning. Memoranda in Computer and Cognitive Science MCCS-95-288. Las Cruces, N.M.: New Mexico State University.

Raskin, Victor, and Sergei Nirenburg 1996. Adjectival modification in text meaning representation. Proceedings of COLING '96. Copenhagen.

Siegel, Muffy E. A. 1976. Capturing the Adjective, Unpublished Ph.D. Thesis, University of Massachusetts, Amherst, MA.

Siegel, Muffy E. A. 1979. Measure adjectives in Montague grammars. In: Steven Davis and Marianne Mithun (eds.), Linguistics, Philosophy, and Montague Grammar. Austin, TX: University of Texas Press, pp. 223-261.

Smadja, Frank 1991. Macrocoding the lexicon with co-occurrence knowledge. In: Uri Zernik (ed.), Lexical Acquisition: Exploiting On-line Resources to Build a Lexicon. Hillsdale, N.J.: Erlbaum, pp. 165-189.

Spang-Hanssen, Ebbe 1990. La sémantique des adjectifs spatieux. Revue Romane 25:2, pp. 292-309.

Spejewski, Beverly 1995. Two classes of frequency adverbs. Paper presented at the LSA Annual Meeting.

Szalay, L. B., and J. Deese 1978. Subjective Meaning and Culture: An Assessment Through Word Association. Hillsdale, N.J.: Erlbaum.

Teller, Paul 1969. Some discussion and extension of M. Bierwisch's work on German adjectivals. Foundations of Language 5, pp. 185-217.

Viegas, Evelyne, Boyan Onyshkevych, Victor Raskin, and Sergei Nirenburg 1996. From submit to submitted via submission: On lexical rules in large-scale lexicon acquisition. In: Proceedings of ACL '96. Santa Cruz, CA: University of California.

Viegas, Evelyne, and Victor Raskin 1996. Lexical Acquisition: Guidelines and Methodology. Memoranda in Computer and Cognitive Science MCCS-96-2xx. Las Cruces, N.M.: New Mexico State University (in preparation).

Zhurinskiy, Alfred N. 1971. O semanticheskoy strukture prostranstvennykh prilagatel'nykh /On the semantic structure of spatial adjectives/. In: Semanticheskaya struktura slova. Moscow: Nauka, pp. 196-24. 